\title{
The Magnetic Tracker with Improved Properties for the Helmet-Mounted Cueing System
}

\author{
Michail Zhelamskij ${ }^{1}$
}

\begin{abstract}
This article highlights both theoretical and experimental experiences in the field of helmet-mounted cueing systems. The current state of these systems is described as optical and hybrid. The adventures of the positioning under local magnetic field are considered, and the directions for further improvement of magnetic technology are identified. A new method is proposed for the local magnetic field creation to increase update rate, to reduce the influence of the Earth's magnetic field, and to reduce energy consumption of helmet-mounted cueing systems in relation to known prototypes. A mathematical model of positioning field is offered. The accuracy of the field mathematical description is studied for different shapes of windings. The transients are investigated in the source of positioning field and in the interior of the cockpit. In addition, a mathematical model of magnetic measurements is proposed, and the main sources of measurement and positioning errors are investigated. The calculation algorithm of the helmet's coordinates is considered based on the results of magnetic measurements. The results of physical models research are given, and the operation of a sample in the full range of angles is shown. The trial mapping is conducted for the field created by the source with a ferromagnetic core. Positioning of the helmet's movement on specified paths is performed, and the results make it possible to figure out the next generation of helmetmounted cueing systems with extended angles range, higher angular and linear accuracy, increased update rate $[200 \mathrm{~Hz}]$, and minimized influence of Earth's magnetic field.
\end{abstract}

KEYWORDS: Helmet, Cueing systems, Magnetic field.

\section{INTRODUCTION}

The helmet-mounted cueing system (HMCS) is a device used in modern combat aircraft, which allows the pilot to designate the on-board weapon and other equipment at the target in accordance with the direction of sight. Before the HMCS appeared, in close combat, the pilot had to align the aircraft to shoot at a target. Using the head angle as a pointer to direct the weapons, the pilot can point his head at the target to actuate a weapon. This enables making more attacks, without having to maneuver to the optimum firing position. These systems allow targets to be designated with minimal aircraft maneuvering, minimizing the time spent in the threat environment and allowing greater lethality, survivability, and pilot situational awareness. These devices were created first in South Africa (Mirage F1, mid-1970s), then in the Soviet Union (MIG-29, 1985), Israel (Python-4, 1990) and, finally, in the United States (AIX-9X missile, 1990) (Melzer, 1997). If the position of the helmet is used to point the missile, it thus must be calibrated and fit securely on the pilot's head. That is why HMCS should be considered from a scientific point of view.

Figure 1 shows the position and orientation of the helmet in the coordinate system of the aircraft. The task of targeting is the orientation calculing of the movable helmet coordinate system $X^{\prime} Y^{\prime} Z^{\prime}$ in relation to the stationary coordinate system $X Y Z$ of aircraft. The line between the pilot's eye and the reticle on the visor is known as line of sight (LOS) between the aircraft and the intended target. The user's eye must stay aligned with the sight direction. To do it, the reticle $R$ should be rigidly connected with the helmet and capture the view direction in

\footnotetext{
三 1.Polytechnical University - Department of Measurement and Technologies - Saint Petersburg - Russia.

Author for correspondence: Michail Zhelamskij | Polytechnical University - Department of Measurement and Technologies | Politekhnicheskaya ul., 29 | 195251 Saint Petersburg - Russia | E-mail: zhelamsk@rambler.ru

Received: 04/08/2016 | Accepted: 06/03/2016
} 
relation to it. Therefore, the pilot's eye always looks at the target through the reticle.

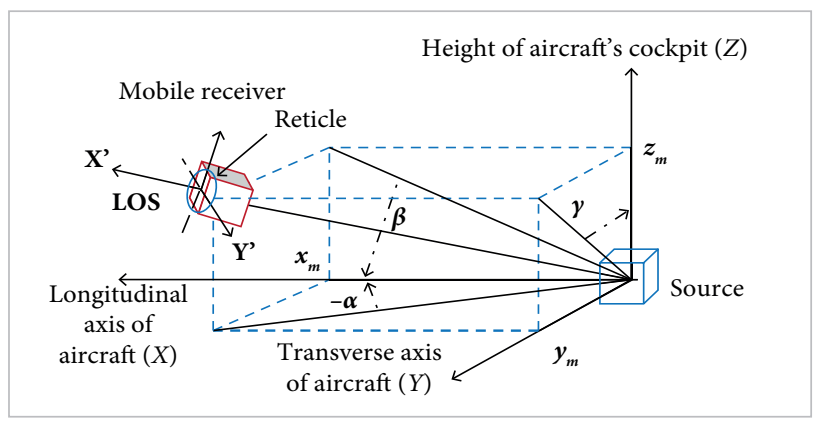

Figure 1. Linear position and orientation of mobile receiver relative to the source in the Cartesian coordinate system.

HMCS design must sense the elevation, azimuth and roll of the pilot's head in relation to the aircraft with sufficient precision even during maneuvering and rapid head movement. The $X^{\prime}$ axis indicates the direction of the target and corresponds to LOS. Azimuth $(a)$ and elevation $(b)$ determine the LOS direction. Linear coordinates $x, y$, and $z$ of the helmet in the cockpit, calculated by any HMCS, can be used to monitor the pilot's status. Information about the roll is useful for stabilizing the image on the helmetmounted display for the accounting of the pilot's vestibular.

The range of the helmet linear motion should not be lesser than $1 \mathrm{~m}$. The precision of cueing, angular error between LOS and derived cue, is determined by the field of view (FOV) of the seeker of the air-to-air missile. The accuracy of the LOS positioning should be much lesser than the FOV of the missile, which is about $1.8^{\circ}$ for an infrared heatseeker (Kopp 1982). The accuracy should be equal over all range of helmet angular motion, and the common field of view of HMCS, angular range over which the sight can still produce a suitably accurate measurement, should be maximum. The latency or slew rate, how much lag there is between the helmet and the cue, should be minimum. The weight of helmet-mounted part of HMCS should be minimum, as well as the power consumption. Otherwise, the update frequency should be maximized. It is enough the linear position coordinates accuracy around centimeters. The roll should be determined with accuracy at the level of units of angular degrees.

Any HMCS includes a movable part, located on the helmet, as well as another item, fixed in the cockpit. Both can be a receiver or a source of local physical fields. The computer, also included in HMCS, solves the mathematical positioning task. The helmet-mounted display will not be considered here.
The new theory is proposed with 6 degrees of freedom (DOF) of magnetic positioning at short distance. The way to organize the local magnetic field is described to improve accuracy of cueing. The mathematic models to create and measure the local positioning field are suggested. The proposal determines a concept of the new generation of magnetic trackers with improved properties. The test results of the first magnetic cueing sample are shown.

The results of numerical simulations of coordinates calculations in the positioning field are shown, besides the results of the mathematical and physical models investigations. The error estimation of the positioning field descriptions and measurements were done as well as positioning ranges concerning angular and linear displacement.

\section{BASIC DEFINITIONS}

It is known the HMCS based on the optical triangulation (Elbit Systems of America ${ }^{\circledR}$ 2016; Buganov 2016; Defencetalk. com 2007). The triad of one-by-one light emitting diodes (LEDs) is located on the helmet's surface. Two fixed on-board receivers are split in the cockpit room. The coordinates of each LED $\left[x_{d}, y_{d}\right]$ on the surface XOY can be obtained from the following system of equations:

$$
\left\{\begin{array}{l}
y_{d}=K_{L}\left(\alpha_{L} \cdot x_{d}+A_{L}\left(\alpha_{L}\right)\right. \\
y_{d}=K_{R}\left(\alpha_{R} \cdot x_{d}+A_{R}\left(\alpha_{R}\right)\right.
\end{array}\right.
$$

where: $\alpha_{L}$ and $\alpha_{R}$ are the bearing on each LED from 2 split receivers, obtained from the measurement; $K_{L}=-\operatorname{tg} \alpha_{L}$; $K_{R}=-\operatorname{tg} \alpha_{R} ; A_{L}=x_{2} \times \operatorname{tg} \alpha_{L}+y_{2} ; A_{R}=x_{1} \times \operatorname{tg} \alpha_{R}+y_{1}$; the coefficients; $x_{1}, y_{1}, x_{2}$, and $y_{2}$ are the coordinates of receivers in the cockpit.

The coordinates of the 3 LEDs together with the dimensions of emitting triad are enough to determine the spatial position of the helmet by the methods of analytic geometry, using the solution of Eq. 1 for each LED. The angular positioning accuracy is at the level of $\delta \varphi \geq 45^{\prime}$. The optical HMCS operates within a cone not greater than $\pm 45^{\circ}$. Accuracy depends on the helmet's orientation because the helmet itself closes the visibility between LED and receivers, and triangulation triangles are degenerated in the line.

A further approach appeared recently to extend the range of operation of the optical tracker, through the integration of helmet-mounted LEDs together with the gyroscopes and accelerometers (Thales Visionix, Inc. 2016; BAE Systems 2016). Hybrid inertial tracking systems employ a sensitive inertial 
measurement unit and optical sensor to provide reference to the aircraft. In the previous operating range, the same optical system is used. Beyond the range of optical tracker, the inertial sensors are used, which have a fundamentally permanent drift of output signals.

Micro-electro mechanical systems (MEMS) contain both gyroscopes and accelerometers and allow to measure full acceleration and orientation of the helmet together with the aircraft movements. It should be taken into account that the full acceleration $(a)$ of the aircraft reaches $10 \mathrm{~g}$ during the maneuvers, whereas the head movement, just $0.01 \mathrm{~g}$. An estimation showed that the modern MEMS like ADIS16448 (Analog Devices 2016) measures the helmet's orientation angles with error at level $\Delta \varphi^{3} \pm 0.3$ angular degree per second for the aircraft acceleration at the level $a=1 \mathrm{~g}$. In the same condition, the linear coordinates of the helmet are measured with the error at the level $\Delta x= \pm 0.22 \times t^{2} \mathrm{~m}$. It is clear that the angular error exceeds requirements for HMCS during the first seconds, and the linear coordinates error reaches the level of the percentage meter. Thus, hybrid tracker allows expanding the ranges of positioning angles only by short-term use of inertial sensor out of operation range of optical HMCS. Common accuracy in the initial angular range is still determined by the worst element - optical tracker.

The magnetic tracking system includes the fixed source of local magnetic field, movable receiver on the helmet and on-board computer, which resolves 3 tasks simultaneously source controlling, magnetic measurements and coordinates calculation. The procedure of active magnetic positioning intends to establish local non-uniform magnetic field with a known spatial distribution, in which the magnetic induction measurement is performed using sensors located at the helmet. The calculation of the position and orientation of a movable helmet-mounted receiver is associated with the solving of systems of non-linear equations, which contain the results of independent measurements of magnetic induction at the point of observation under specified parameters of the field source (the right parts) and unknown linear and angular coordinates of receiver in space of the positioning field source (the left parts of equations).

The known active magnetic trackers are based on alternating current (AC) with sinusoidal shape (Fig. 2; Raab 1977) or direct current (DC) with pulse shape (Fig. 3; Blood 1989) local magnetic field.

The tracker includes 3 orthogonal windings in the fixed source of local magnetic field and 3 orthogonal sensors in the mobile receiver. In the first case (AC), all windings of the source work simultaneously $\left(I_{x}+I_{y}+I_{z}\right)$ on different frequencies. In the second case, they operate in the pulse mode, one by one, in sequence: $I_{x} \rightarrow I_{y} \rightarrow I_{z}$. The pause $\left(t_{3}-t_{4}\right)$ is designed to take into account the Earth's magnetic field (EMF), whose influence is subtracted from each of the measured signals, obtained from the windings.

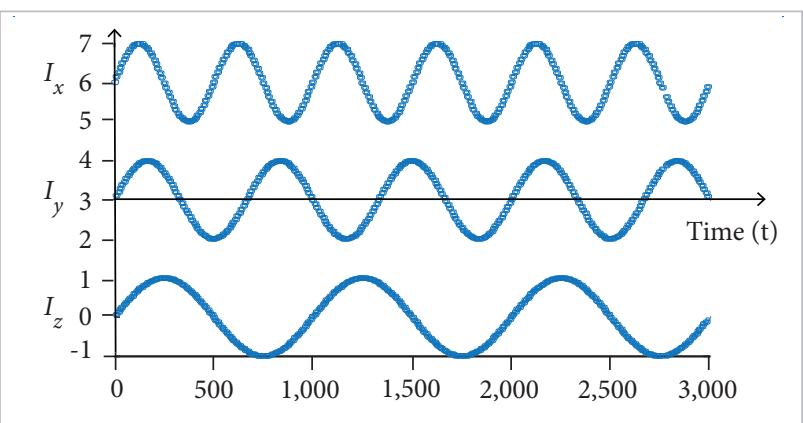

Figure 2. Dimensionless charts of the excitation currents through the windings of the sinusoidal magnetic positioning systems.

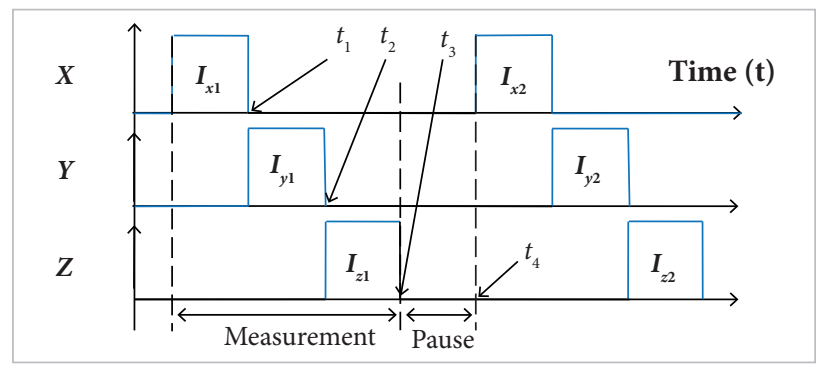

Figure 3. Dimensionless charts of the excitation currents in pulse magnetic positioning systems.

In both cases, the coordinates of the helmet are determined from the following equation system solution:

$$
B_{x} \cdot \cos \varphi+B_{y} \cdot \cos v+B_{z} \cdot \cos \xi=B_{x x}
$$

where: $B_{x}, B_{y}$, and $B_{z}$ are the computed values of axial components of magnetic fields induction vector, defined by mathematical models of positioning field, at coordinates of the initial approximation $x_{s}, y_{s}$, and $z_{s} ; \cos \varphi, \cos v$, and $\cos \xi$ are the values of guide cosines of Hall sensor in a fixed coordinate system; $B_{x x}$ is the measured value of the induction of one sensor from one source winding. Designating $B_{x} \times \cos \varphi+B_{y} \times \cos v$ $+B_{z} \times \cos \xi=F$ and $B_{x x}=\Theta$, we get:

$$
F(\psi, X)=\Phi-\Theta=0
$$


where: $\psi=\left[\mathrm{M}, t, X_{c}, T, C_{0}, I_{H G}, I_{S}, \Delta \mathrm{MS}, w\right]$ is the vector of parameters included in the mathematical model of establishment and measurement of positioning field, described in Eq. 9. The number of equations $M$ in Eq. 3 shall not be less than the number of the desired coordinates $M \leq N \times K$, where $N$ is the number of windings in the source and $K$ is the number of sensors in the receiver. The calculation of the 6 coordinates is reduced to the solution of systems (Eq. 3), containing nonlinear equations (Eq. 2). In this case, we have: $\mathbf{F}(\mathbf{X}, \psi)=0$, where $\mathbf{F}$ is the vector function of $\mathbf{X}$ - vector of intended coordinates at the observation point $\mathbf{X}=(x, y, z, \alpha, \beta, \gamma)$ :

$$
\vec{F}(X, \Psi)=\left[\begin{array}{c}
F_{I}(x, y, z, \alpha, \beta, \gamma, \Psi) \\
\ldots \ldots \ldots \ldots \ldots \ldots \ldots \ldots \\
F_{6}(x, y, z, \alpha, \beta, \gamma, \Psi)
\end{array}\right]=0
$$

where: $\gamma$ is the roll angle.

The desired linear coordinates of mobile receiver $\left[x_{m}, y_{m}, z_{m}\right]$, recorded in Eq. 2 as arguments to the mathematical description of the axial component of induction vector, $\mathbf{B}_{m}=\left[B_{x}\left(x_{m}, y_{m}, z_{m}\right), B_{y}\left(x_{m}, y_{m}, z_{m}\right), B_{z}\left(x_{m}, y_{m}, z_{m}\right)\right]^{\mathrm{T}}$, and orientation angles of the receiver, $\left[\alpha_{m}, \beta_{m}, \gamma_{m}\right]$, are present in guide cosines through the matrix of the movable receiver rotation: $A_{x y z} \equiv A_{x}(\alpha) \times A_{y}(\beta) \times A_{z}(\gamma)$. Thus, the system (Eq. 4), composed of 6 equations, has a strictly non-linear nature and can only be solved by numerical methods of iterative approximation.

Figure 4 shows a typical layout of magnetic HMCS in the cockpit through the example of the project Vista for the F-16D aircraft (Merryman 1994). A similar layout has another magnetic HMCS (Elbit Systems 2006; Kopp 1998; Thales Group 2016). Still in Fig. 4, there is a cubic source of the local magnetic positioning field, established behind the pilot's right shoulder. The movable receiver is hidden behind the external surfaces of

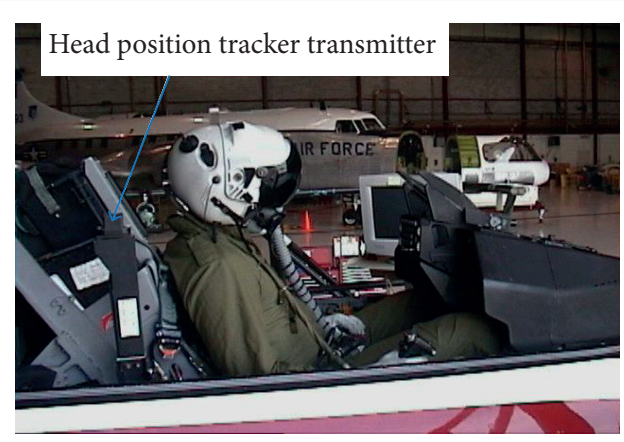

Figure 4. Magnetic positioning system of Vista layout aboard the F-16D. the protective helmet. The main advantage of magnetic methods of positioning is that the LOS is not required between source and receiver. Therefore, the helmet cannot influence the ranges of operation due to transparency for the stationary magnetic field. This advantage sets out a broad range of the operation angles for magnetic HMCS. As a result, the operation distance - up to $1 \mathrm{~m}-$ is comparable with the cockpit size, and the angular range theoretically varies up to $\pm 180^{\circ}$. Positioning accuracy of the magnetic HMCS is better than that of the optical one and declared at the level $\delta X \leq \pm 1 \mathrm{~mm}$ and $\delta \varphi \leq \pm 0.5$ angular degree for up to $1-\mathrm{m}$ distance for the stationary receiver with unchanged orientation.

Dielectric interior elements have no effect on the positioning accuracy for the magnetic method. Electrically conductive materials have effect for AC method only, depending on the size and distance between source, receiver and element. The mapping of the influence of eddy currents on the AC method is labor-intensive (Lescourret 1997) and not always yields the result, particularly for helicopters with cramped cockpits. The practice has shown that AC method cannot be used in helicopters (Egli et al. 1983). Conductive interior elements do not affect the DC method when a certain duration of the magnetic field pulse is selected (see next). The magnetic materials have effect on both AC and DC magnetic methods, but the magnetic elements in the cockpit interior are much lesser than the electrically conductive materials. Therefore, only DC method will be considered further.

The EMF vector is added to the field positioning. A contribution of EMF depends on the ratio between the object velocity and the update frequency and, for stationary object, it is zero. For DC method, the EMF is taken into account one time per full cycle of the pulse field switching. The magnetic field switches to 4 times faster than the output updated, as shown in Fig. 3. This is why the update frequency should be increased more over the current $100 \mathrm{~Hz}$ limit to reduce the EMF contribution.

The main parameters of AC and DC trackers, available in the literature, are given in Table 1. The main sources of measurement errors of the positioning field are shown in Zhelamskij (2014b), among which the influence of the sensors' size near the source is dominated as well as their spatial separation and the accuracy of the mathematical description of the positioning field. The sensors' size reaches $4-5 \mathrm{~mm}$ in modern aviation trackers (Kuipers 1975), which leads to increased measurement error of module induction vector near a source up to $10 \%$. Therefore, the decreasing in the sensors' size is actual and accounts for their 
spatial separation, as described next. The already mentioned aircraft trackers prototypes use either inductive sensors in AC method or fluxgate sensor in DC one. In both cases, there are induction measuring windings with large area, which is sensitive to high-frequency interference. Therefore, when the sensitivity of measurements is at the level of 10 nTesla, the sustainability of mobile receiver to external disturbances, for example, radar's radiation, is relevant. A power consumption should be minimized for an on-board equipment in any case. The task is to find an opportunity to do it. Now we have about $100 \mathrm{~W}$ per sphere with $1-\mathrm{m}$ radius. There is important to reduce the value of power consumption for on-board cueing system.

Table 1. Comparison of parameters of the prototypes (Polhemus 2016; Ascension Technology Corporation 2016).

\begin{tabular}{|c|c|c|c|}
\hline Title & $\begin{array}{c}\text { Sourcereceiver } \\
\text { maximum } \\
\text { distance }(\mathrm{m})\end{array}$ & $\begin{array}{c}\text { Update } \\
\text { frequency } \\
{[\mathrm{Hz}]}\end{array}$ & $\begin{array}{c}\text { Static accuracy } \\
\text { at the distance } \\
\text { of } 0.78 \mathrm{~m}\end{array}$ \\
\hline $\begin{array}{c}\text { Polhemus } \\
\text { "Patriot" }\end{array}$ & 1.5 & 60 & $\pm 1.5 \mathrm{~mm} ; \pm 0.4^{\circ}$ \\
\hline $\begin{array}{c}\text { Ascension } \\
\text { "DriveBAY" }\end{array}$ & 0.78 & $>120$ & $\pm 1.4 \mathrm{~mm} ; \pm 0.5^{\circ}$ \\
\hline
\end{tabular}

The above review clearly showed that the pulse magnetic method of positioning is the most promising among others concerning the range of operation, precision, and interior influence. However, it can be seen that the existing magnetic tracking technology does not fully meet these requirements. Thus, this paper describes the results of an investigation of the possibility to improve the magnetic trackers for cueing task. It must be explored the possibility of reducing power consumption of the HMCS as well as the EMF's influence. It is necessary to increase the update frequency when possible and to increase the immunity HMCS against the external radar. Concerning the mapping, it can be supposed that this procedure is not needed, because the magnetic elements on-board the light modern airplane or helicopter are much smaller than the electrically conductive material. However, the movable ferromagnetics should not be situated inside the zone of the helmet movement. An effect of electroconductivity elements for DC method is determined by the ratio between magnetic field pulse duration and eddy current decay time constant. Therefore, the eddy current in the cockpit interior should be investigated. The main objective of this article is to declare the goals for a next generation of magnetic helmet-mounted cueing system with improved parameters as update frequency, EMF influence, power consumption, and outer noise immunity. The new mathematical models to create and measure the positioning magnetic field are presented. The advantages of the new method achieved in comparison with the prototype are described, the estimations of the eddy currents influence are given, and the algorithm of iterative calculation of coordinates of the mobile receiver is considered.

\section{METHODOLOGY}

An investigation of new magnetic cueing system was fulfilled with the methods described next, such as mathematic description and simulation, system analysis, comparison with known theories, physical modeling of non-standard elements of the system, and the actual movement of the mobile receiver at the final stage of positioning modeling.

To satisfy the formulated requirements, a new method is proposed to organize the positioning local magnetic field, and investigations are performed. The new method is theoretically considered as a means of reducing the impact of EMF and power consumption. A mathematical model of the positioning field is proposed. The investigation of the transition process is performed, as well as of the accuracy of the field descriptions in the presence of conductive elements. The mathematical model of movable receiver is also proposed and investigated regarding both measurement errors and eliminating the interference from on-board network and external sources. They are explored in different approaches for the iterative solving of the non-linear equations systems, linking the results of magnetic measurements with the desired coordinates. An investigation of the physical models allowed to estimate the mathematical description accuracy of the source with and without ferromagnetic core. Besides, the metrological research of movable receiver was performed. The 6-DOF tracking was fulfilled when the receiver was moving at the specified path.

A new (the 3rd in the world) method to organize the local magnetic positioning field is called alternating-direct current (ADC) method (Zhelamskij 2011). The bipolar current pulses are offered in the new method, running consecutively one by one without pause. Figure 5 shows the comparative chart of pulse currents in the source windings for DC prototype, separated by a pause, and for the new ADC method. The top graph is combined for 3 windings of DC method. Three lower graphics are separated for each of the windings of ADC method. In both 


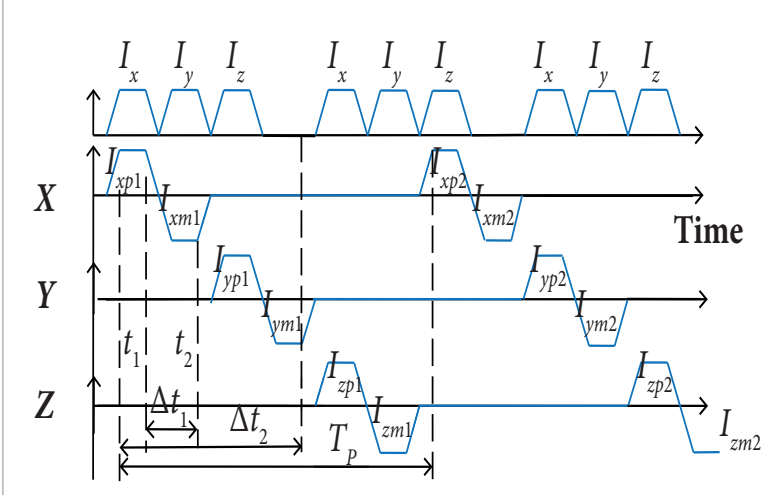

Figure 5. Schematic comparison of ADC and DC methods to organize the field of positioning.

cases, the graphics are post-poned for 3 orthogonal windings of local positioning systems (Raab 1977; Blood 1989), where the magnetic field pulses duration is equal. The graphics are schematically shown. Actually, the rise and fall of pulse current is much lesser than the pulse duration. At the top of each bipolar impulse, the positioning field vector is folded with the EMF at a mobile receiver:

$$
\overrightarrow{\mathrm{B}}_{\mathrm{m}}=\overrightarrow{\mathrm{B}}_{\mathrm{EMF}}+\overrightarrow{\mathrm{B}}_{\mathrm{P}}
$$

where: $\vec{B}_{\mathrm{m}}$ is the total induction vector, measured by sensors of the mobile receiver; $\vec{B}_{E M F}$ is the EMF vector; $\vec{B}_{P}$ is the vector of positioning field.

One can write, from Eq. 5 , the system of equations for time points $t_{1}$ and $t_{2}$, separated by an interval $\Delta t_{1}$, as shown in Fig. 5:

$$
\left\{\begin{array}{l}
\overrightarrow{\mathrm{B}}_{\mathrm{EMF}}\left(\mathrm{t}_{1}\right)+\overrightarrow{\mathrm{B}}_{\mathrm{P}}\left(\mathrm{t}_{1}\right)=\overrightarrow{\mathrm{B}}_{\mathrm{m}}\left(\mathrm{t}_{1}\right) \\
\overrightarrow{\mathrm{B}}_{\mathrm{EMF}}\left(\mathrm{t}_{2}\right)-\overrightarrow{\mathrm{B}}_{\mathrm{P}}\left(\mathrm{t}_{2}\right)=\overrightarrow{\mathrm{B}}_{\mathrm{m}}\left(\mathrm{t}_{2}\right)
\end{array}\right.
$$

This system can be resolved relatively to the vector of the positioning field $\overrightarrow{\mathrm{B}}_{\mathrm{P}}$. Subtracting the above equations from each other and assuming equality of currents through the source winding $|I(+)|=|I(-)|$ at the moments $t_{1}$ and $t_{2}$, one can write the common solution of the system (Eq. 6):

$$
\mathrm{B}_{\text {meas }}=2 \overrightarrow{\mathrm{B}}_{\mathrm{m}}=\left\lfloor\overrightarrow{\mathrm{B}}_{\mathrm{P}}\left(\mathrm{t}_{1}\right)+\overrightarrow{\mathrm{B}}_{\mathrm{P}}\left(\mathrm{t}_{2}\right)\right\rfloor+\left\lfloor\overrightarrow{\mathrm{B}}_{\mathrm{EMF}}\left(\mathrm{t}_{1}\right)-\overrightarrow{\mathrm{B}}_{\mathrm{EMF}}\left(\mathrm{t}_{2}\right)\right\rfloor
$$

The residual value of the EMF, $\left.\Delta \mathrm{B}_{\mathrm{EMZ}}=\overrightarrow{\mathrm{B}}_{\mathrm{EMF}}\left(t_{1}\right)-\overrightarrow{\mathrm{B}}_{\mathrm{EMF}}\left(t_{2}\right)\right]$, from Eq. 7, depends on the angular velocity $\omega$ of the object and the time interval $\Delta t_{1}=t_{2}-t_{1}$. The value $\Delta B_{E M Z}$ is zero for the stationary object, when $\omega=0$.
Hence, taking into account $\Delta t_{2} \geq 2 \Delta t_{1}$, the use of bipolar positioning field allows to double the amplitude of measured induction versus DC method and reduce twice the impact of EMF:

$$
\mathrm{B}_{\text {meas }}=2 \mathrm{~B}_{\mathrm{p}}, \Delta_{\mathrm{BEMFADC}} \leq \frac{1}{2} \Delta \Delta_{\mathrm{Bm}} \mathrm{FPC} .
$$

Besides, the condition $\Delta t_{2} \geq 2 \Delta t_{1}$ means twice-increased update rate versus the prototype.

\section{POWER CONSUMPTION FOR ON-BOARD HMCS}

The averaged power consumption for DC method $\left(P_{1}\right)$ and ADC one $\left(P_{2}\right)$ is compared as follows:

$$
\frac{P_{1}}{P_{2}}=\frac{\frac{1}{t_{p}} \int_{0}^{t_{p}=4 T} I_{x}^{2} R d t}{\frac{1}{t_{p}} \int_{0}^{t_{p}=6 T} I_{x p m}^{2} R d t}
$$

where: $R$ is the full resistance of winding; $t_{p}$ is the full period of the windings switching; $I_{x}$ and $I_{x p m}$ are the pulse current amplitudes for DC and ADC methods, respectively; $T$ is the duration of one unipolar pulse of positioning field, which is equal for both DC and ADC methods.

It can be concluded from Eq. 8:

- If the amplitudes of pulsed current are equal $\left(I_{x}=I_{x p}=I_{x m}\right)$, we have $\left(P_{1} / P_{2}\right) /(3 / 4)$ (Fig. 6, mode $\left.A\right)$.

- If the sweep of measured inductions is equal $\left(B_{D C}=2 B_{A D C}\right)$, we have $\left(P_{1} / P_{2}\right) / 3$ (Fig. 6, mode $B$ ).

- If the root mean square (RMS) of noise is given ( $\sigma_{N}=$ constant), then the signal-noise ratios for the prototype $\left(\mathrm{SNR}_{1}=\mathrm{B}_{1} / \sigma_{N}\right)$ and for the bipolar fields $\operatorname{method}\left(\mathrm{SNR}_{2}=2 \cdot \mathrm{B}_{2} / \sigma_{N} \cdot \sqrt{2}\right)$ are identical $\left(\mathrm{SNR}_{1}=\mathrm{SNR}_{2}\right)$ for twice-reduced power consumption $\left(P_{1} / P_{2}=2\right)$, as follows from the calculations (Fig. 6, mode $C$ ):

$$
\frac{B_{1}}{\sigma_{N}}=\frac{2 \cdot B_{2}}{\sigma_{N} \cdot \sqrt{2}}, I_{2}=I_{1} \cdot \frac{\sqrt{2}}{2}, P_{2}=P_{1} \cdot \frac{1}{2}
$$

The comparison of the parameters of DC and ADC methods is presented in Table 2. The module of induction vector at a great distance from the windings with current can be described by the following simplified formula: $B_{M} \approx \Psi / R^{3}$, where $\Psi$ is the function that describes the effect of the winding sizes 
and the flowing current. In accordance with Fig. 6, for the same currents, the modules values used to calculate the coordinates for the prototype $\left(B_{1}\right)$ and for the new method $\left(B_{2}\right)$, which differ by half, will be read as $B_{1}=\Psi / R_{1}^{3}$ and $B_{2}=2 \Psi / R_{2}^{3}$. Equating $B_{1}=B_{2}$, we get $\left(R_{2} / R_{1}\right)^{3}=2$ or $R_{2}=1.25 \times R_{1}$.

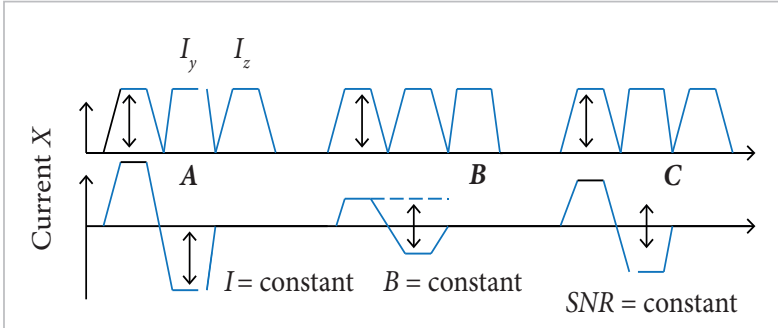

Figure 6. Three modes of ADC method operation.

Table 2 shows that the new proposed ADC method provides increased positioning zone radius on $25 \%$, doubles the update rate and the magnitude of measured induction, at least half the impact of EMF, and, up to factor 3, reduces the power consumption. A slight increase in latency can be off-set by the application of Kalman filter, performing "forward extrapolation".

Table 2. Comparison of parameters of the prototype and the new method.

\begin{tabular}{|c|c|c|}
\hline Parameter & DC & ADC \\
\hline $\begin{array}{c}\text { Update frequency for } \\
\text { same pulse duration }\end{array}$ & $F$ & $2 F$ \\
\hline $\begin{array}{c}\text { Measured inductance } \\
\text { deviation }\end{array}$ & $\Delta B$ & $2 \Delta B$ \\
\hline $\begin{array}{c}\text { Coverage zone radius } \\
\text { Latency }\end{array}$ & $R$ & $1.25 R$ \\
\hline Power consumption & $\mathrm{P}$ & $\begin{array}{c}0.33 P(\Delta B=\text { constant }) \\
0.5 P(\mathrm{SNR}=\text { constant })\end{array}$ \\
\hline Earth's field influence & $4 \mathrm{~T}$ & $2 \mathrm{~T}$ \\
\hline
\end{tabular}

Due to the presented advantages, it can be verified a new way to organize the positioning field, because it better meets the conditions for HMCS as update rate, power consumption, and the impact of EMF.

\section{MATHEMATICAL MODEL}

To describe the spatial distribution of the positioning field, an expression for the induction vector projection can be written and is created at observation point with coordinates $x_{m}, y_{m}$, and $z_{m}$ by one of the positioning field source windings on the normal to the sensor surface in the mobile receiver with orientation angles like $\alpha, \beta$, and $\gamma$ (Fig. 1):

$$
B_{m}\left(x_{m}, y_{m}, z_{m}, \alpha, \beta, \gamma, t\right)=\Phi(X, M)=F_{1}\left(x_{m}, y_{m}, z_{m}\right)
$$

where: $F_{1}\left(x_{m}, y_{m}, z_{m}\right)=\left[B_{x}\left(x_{m}, y_{m}, z_{m}, A, B, I\right), B_{y}\left(x_{m}\right.\right.$, $\left.\left.y_{m}, z_{m}, A, B, I\right), B\left(x_{m}, y_{m}, z_{m}, A, B, I\right)\right]^{\mathrm{T}}$ is the function that defines the dependency between the induction vector and the linear coordinates; $A$ and $B$ are the side lengths of the rectangular winding; $F_{2}(\alpha, \beta, \gamma)=S_{1 r} \times A_{x y z}$ is the function of the sensor orientation influence on the measured value, being $A_{x y z} \equiv A_{x}(\alpha) \times A_{y}(\beta) \times A_{z}(\gamma)$ the full rotation matrix on 3 angles in the sequence $\alpha \rightarrow \beta \rightarrow \gamma$ and $A_{x}(\alpha), A_{y}(\beta)$, and $A_{z}(\gamma) 3$ matrices with size $3 \times 3$, reflecting the coordinate transformation for the sequential object rotation around each axis of the stationary $3-D$ Euclidean space $X Y Z ; F_{3}(t)$ is the function that specifies the sequence of the independent fields over time, as illustrated in Fig. 3, respectively, for the winding $X \rightarrow Y \rightarrow Z$ :

$$
\begin{aligned}
& F_{3 X}(t)=\left\{\begin{array}{c}
+1 \\
-1 \\
0
\end{array}\right\} \text { for }\left\{\begin{array}{c}
0<t<T \\
T<t<2 T, \\
2 T<t<6 T
\end{array}\right. \\
& F_{3 Y}(t)=\left\{\begin{array}{c}
0 \\
+1 \\
-1
\end{array}\right\} \text { for }\left\{\begin{array}{c}
0<t<2 T, 4 T<t<6 T \\
2 T<t<3 T \\
3 T<t<4 T
\end{array},\right. \\
& F_{3 Z}(t)=\left\{\begin{array}{c}
0 \\
+1 \\
-1
\end{array}\right\} \text { for }\left\{\begin{array}{c}
0<t<4 T \\
4 T<t<5 T \\
5 T<t<6 T
\end{array}\right.
\end{aligned}
$$

where: $T$ is the half period of bipolar pulse $T=\Delta t_{1}=t_{2}-t_{1}$ in Fig. 5.

The components of the induction vector included in factor $F_{1}$ of Eq. 9 are fully recorded in Zhelamskij (2014a, 2015) for flat rectangular coils, which have a magnetic moment oriented on each axis of the stationary coordinate system $X Y Z$ of aircraft from Fig. 1.

A given model (Eq. 9) allows to explore a positioning field in different conditions, as the shape and size of windings, their mutual location and orientation, orientation of mobile receiver, the frequency of the switching and transients, and influence of electrically conductive and ferromagnetic material on the 
tracking accuracy. The result of the simulation will allow verifying requirements for the elements of the distributed source.

To estimate the accuracy of the mathematical model, in any cases, the amendments $\Delta B=\left(B_{C}-B_{M}\right) \in[R]$ were investigated within the positioning zone $R$, where $B_{C}$ and $B_{M}$ are, respectively, the calculated and measured values of the induction vector components. At stage of numerical simulation for a coreless source as $B_{M}$, the values from Eq. 9 were used, written for single thin frame with current. In this case, the value of $B_{C}$ described the result of the calculation for ultimate windings cross-section with different shapes. For the source with ferromagnetic core, the calculation was performed also in accordance with Eq. 9, but measured values $B_{M}$ were taken from the mapping results for the helmet movement zone. As a result, the arrays of the amendments to the calculated values were obtained, which are used to modify Eq. 2:

$$
\begin{aligned}
\left(B_{x}+\Delta B_{x}\right) \cdot \cos \varphi & +\left(B_{y}+\Delta B_{y}\right) \cdot \cos v+ \\
& +\left(B_{z}+\Delta B_{z}\right) \cdot \cos v=B_{m 1}
\end{aligned}
$$

where: $B_{x}, B_{y}$, and $B_{z}$ are the calculated values of axial components of the induction vector from Eq. 9; $\Delta B_{x}, \Delta B_{y}$, and $\Delta B_{z}$ are the amendments from the mapping results; $B_{m 1}$ is the full measured value of induction; $\cos \varphi, \cos v$, and $\cos v$ are the guide cosines of the normal to the sensor surface from Eq. 2.

\section{EDDY CURRENTS}

Time dependence of the pulsed current $I_{0}(t)$ through the source windings from Eq. 10 may be written in detail in a mathematical model (Eq. 9) as follows:

$$
\begin{aligned}
& F_{3}(t) \approx I_{0}(t)=-(-1)^{N} . \\
& \left\{\begin{array}{l}
I_{0} \cdot \frac{t}{\tau_{s}}, \text { if } T_{1}^{+}+\frac{T_{n}}{2} \cdot(N-1)<t<T_{2}+\frac{T_{n}}{2} \cdot(N-1) \\
I_{0^{\prime}} \text { if } T_{2}+\frac{T_{n}}{2} \cdot(N-1)<t<T_{4}+\frac{T_{n}}{2} \cdot(N-1), \\
I_{0} \cdot e^{-t / \tau}, \text { if } T_{4}+\frac{T_{n}}{2} \cdot(N-1)<t<T_{5}+\frac{T_{n}}{2} \cdot(N-1)
\end{array}\right.
\end{aligned}
$$

where: $N$ is the number of half periods in contiguous sequence of bipolar current pulses in each measuring channel; $\tau_{s}$ and $\tau$ are the winding current charge and discharge time constants, respectively; $T_{\Pi}$ is the full duration of bipolar current period;
$\left(T_{1}-T_{2}\right)$ represents the front duration; $\left(T_{2}-T_{4}\right)$ is the plateau duration; $\left(T_{4}-T_{5}\right)$ is the fall pulse duration.

The source design should provide the duration of front and fall of the pulse magnetic field much less than plateau duration $\left[\left(T_{1}-T_{2}\right) » t\right]<<\left(T_{2}-T_{4}\right)$.

The mathematical model is created to investigate the influence of eddy currents on the accuracy of the positioning field measurement. Figure 7 shows a single winding $W_{1}$ of the positioning field source, oriented along the $X$ axis of the fixed coordinate system $X O Y$; helmet-mounded receiver $R$ at the observation point with coordinates $x_{m}, y_{m}, z_{m}$; flat conductive element (EL) with radius $r$; linear coordinates of the center $x_{d}, y_{d}$, $z_{d}$; and direction of the normal to the surface of the element as a matrix of guide $\operatorname{cosines~} \mathrm{NM}=[\cos \varphi, \cos v, \cos v]^{\mathrm{T}}=[0,1,0]^{\mathrm{T}}$, which reflects the properties of the helicopter cockpit covering $\left(\Delta=2 \mathrm{~mm}, \rho=2.7 \times 10^{-8} \Omega \mathrm{m}\right)$.

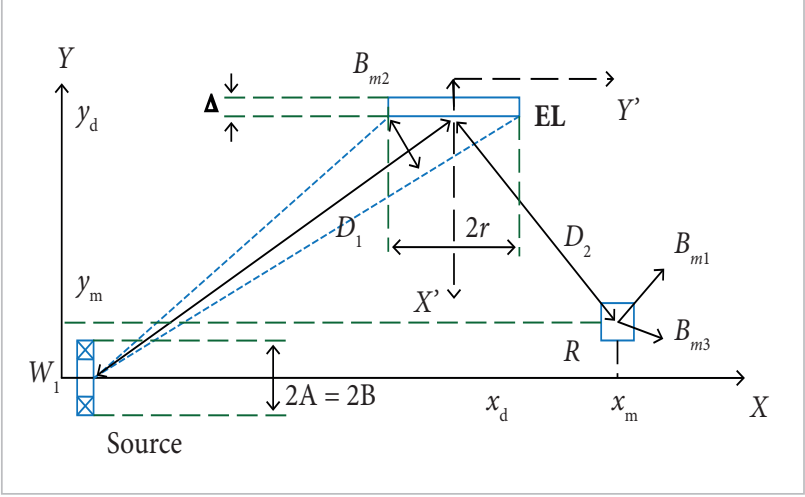

Figure 7. Design scheme of accounting effects of eddy currents on the interior.

Setting the current through the winding $I_{x}$ and rectangular winding dimensions $2 \mathrm{~A}$ and $2 \mathrm{~B}$, the mathematical models of the positioning field are: $B_{X X}=F X X\left(A, B, I_{x}, x, y, z\right), B_{X Y}=F X Y(A$, $\left.B, I_{x} x, y, z\right), B_{X Z}=F X Z\left(A, B, I_{x}, x, y, z\right)$.

The axial components and module of induction vector $B_{m 1}$, generated by the winding at the movable receiver, as well as on the surface of the conductive element, $B_{m 2}$, were calculated from Eq. 9 as shown in Fig. 7. Then, one can estimate the amount of additional field at the receiver $B_{m 3}$, induced by eddy current in the interior EL. The model allows to estimate the ratio $B_{m 1} / B_{m 3}$ depending on the coordinates of the observation point and the center of the EL disc for different $r, \Delta, \rho$, and NM. The used formula (Khalfin 2004) for additional field on the receiver generated by eddy currents in single element of cockpit interior is: 
$B e \sim K_{1} \cdot S /\left(D_{1}^{3} \cdot D_{2}^{3}\right)$

where: $K_{1}$ is the factor to take into account the conductive properties of the element EL; $S$ is the projection of the element EL square, visible from the winding $W_{1}$ (see Fig. 7); $D_{1}$ is the distance between EL and the winding; $D_{2}$ is distance between $\mathrm{EL}$ and the receiver.

\section{MAGNETIC INTERFERENCE}

It is important to take into account the magnetic interference from an on-board network of $400 \mathrm{~Hz}$, which can reach $10-15 \%$ of the amplitude of the positioning field. To implement a differencing method of compensation, it is necessary that the duration of half of the bipolar pulse positioning field $\left(t_{2}-t_{1}\right)$ be a multiple of the total duration of the periods $n \cdot T_{N}$ of the interference from the on-board network: $n \cdot T_{N}=\left(t_{2}-t_{1}\right)=\left(t_{3}-t_{2}\right)=\left(t_{4}-t_{3}\right)=\ldots$, as shown in Fig. 8 for $n=2$. In this case, $t_{2}-t_{1}=5 \mathrm{~ms}$ and $f=100 \mathrm{~Hz}$. Correspondingly, for $n=1, t_{2}-t_{1}=2.5 \mathrm{~ms}$ and $f=200 \mathrm{~Hz}$; for $n=3, t_{2}-t_{1}=10$ $\mathrm{ms}$ and $f=50 \mathrm{~Hz}$. Considering $\mathrm{B}_{\mathrm{EMF}}$ constant and rewriting Eq. 2 in view of the contribution of interference from on-board network in case $t_{2}-t_{1}=2 n / 2 f_{400}$, it is possible to obtain:

$$
\left\{\begin{array}{l}
\vec{B}_{400}\left(t_{1}\right)+\vec{B}_{P}\left(t_{1}\right)=\vec{B}_{m}\left(t_{1}\right) \\
\vec{B}_{400}\left(t_{2}\right)-\vec{B}_{P}\left(t_{2}\right)=\vec{B}_{m}\left(t_{2}\right)
\end{array}\right.
$$

After subtracting the equations, we have:

$$
\begin{aligned}
\mathrm{B}_{\text {meas }} & =\left(\overrightarrow{\mathrm{B}}_{\mathrm{m}}\left(\mathrm{t}_{1}\right)-\left(\overrightarrow{\mathrm{B}}_{\mathrm{m}}\left(\mathrm{t}_{2}\right)\right)=\left[\overrightarrow{\mathrm{B}}_{\mathrm{P}}\left(\mathrm{t}_{1}\right)+\overrightarrow{\mathrm{B}}_{\mathrm{P}}\left(\mathrm{t}_{2}\right)\right]+\right. \\
& +\left[\overrightarrow{\mathrm{B}}_{400}\left(\mathrm{t}_{1}\right)-\overrightarrow{\mathrm{B}}_{400}\left(\mathrm{t}_{2}\right)\right] \rightarrow 2 \overrightarrow{\mathrm{B}}_{\mathrm{m}}
\end{aligned}
$$

It follows from Eq. 14b that the difference result does not contain the influence of magnetic interference from the on-board network. The on-board power supply frequency $f=400 \mathrm{~Hz}$ is not as stable as in terrestrial networks of $50 / 60 \mathrm{~Hz}$ and depends on the aircraft speed engine. Therefore, the duration of the magnetic field pulse floats and strictly corresponds to the selected number of on-board network half period, using the hardware synchronization as shown in the lower graph of Fig. 8 .

\section{ACCURACY}

New modern magnetometers with Hall sensors appeared recently, with the minimum weight of the units gram and the size of a millimeter level (Asahi Kasei Corp. 2016; Ivensense ${ }^{\circledR}$ 2016;

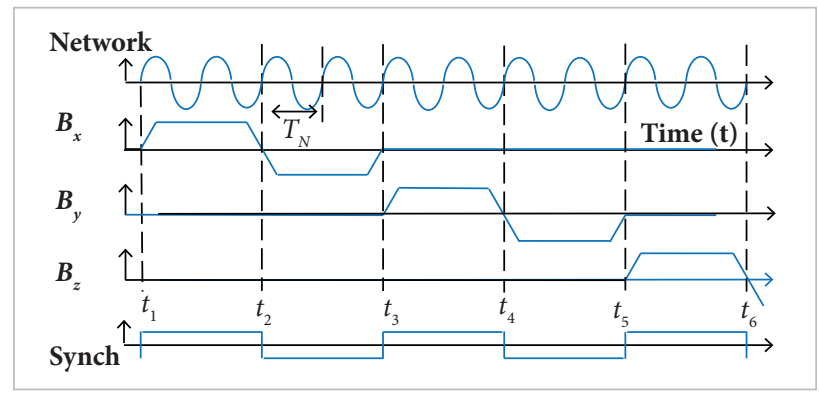

Figure 8. Compensation of magnetic interference influence from the on-board network.

Bosh Sensortec 2016). It is possible to evaluate the possibility of creating the helmet-mounted receiver on such magnetometers that would eliminate the problem of helmet's weight. The immunity of such a device against external interference should be considered as well.

These magnetometers are intended to measure homogeneous EMF only. The mathematical model proposed to apply a magnetometer to measure the inhomogeneous positioning field (Zhelamskij 2014b) is:

$$
\begin{aligned}
U_{\text {out }}(t) & =\left\{K _ { M } \cdot \left[B_{M T}(x, y, z, \alpha, \beta, \gamma, t)+\Delta B_{E M T}(t)+\right.\right. \\
& \left.\left.+\Delta B_{F}(x, y, z)\right] \cdot\left(1+\sum \varepsilon_{s k}(x, y, z)\right)\right\}+\quad(15) \\
& +U_{\text {shift }}(t, T)+U_{R A N D}(t)
\end{aligned}
$$

where: $K_{M}=G(f) \times \operatorname{MS}(T=$ constant $\pm \Delta T) \times I_{H G} \times I_{S}$ is the function which takes into account the sensitivity of the sensor MS, an amplification $G(f)$ of the measuring channel, the excitation currents through the sensors $I_{H G}$ and field source $I_{S}$; $B_{M T}=F_{1}\left(x_{m}, y_{m}, z_{m}\right) \times F_{2}(\alpha, \beta, \gamma) \times F_{3}(t)$ is the projection of the induction vector of positioning field from Eq. $14 \mathrm{~b}$ to be measured; $\sum_{\mathrm{k}=1}^{8} \varepsilon_{\text {sk }}(\mathrm{X}, \mathrm{Y}, \mathrm{Z})$ is the total systematic measurement error, being $\varepsilon_{\mathrm{s} 1}$ the effect of spatial diversity of the receiver's sensors, $\varepsilon_{\mathrm{s} 2}$ the effect of temperature, $\varepsilon_{\mathrm{s} 3}$ the mutual non-orthogonality of the sensors, $\varepsilon_{\mathrm{s} 4}$ the effect of sensors' excitation current, $\varepsilon_{\mathrm{s} 5}$ the effect of source excitation current, $\varepsilon_{\mathrm{s} 6}$ the sensitivity uncertainty, $\varepsilon_{\mathrm{s} 7}$ the error of ADC, $\varepsilon_{\mathrm{s} 8}$ the influence of sensors' finite size; $\Delta B_{E M F}(t)$ is the residual impact of the EMF; $\Delta B_{F}(x, y, z)$ is the accuracy of the mathematical description of the positioning field; $U_{\text {shift }}(t, T=$ constant $\pm \Delta T)$ is the off-set of sensor output voltage; $U_{R A N D}(t)$ is a random process, modeling its own noise of measurement device.

It can be seen from Eq. 15 that the total induction is $B_{S}=B_{M T}(x, y, z, \alpha, \beta, \gamma, t)+\Delta B_{E M F}(t)+\Delta B_{F}(x, y, z)$, applied to 
the sensor, being converted to the measured voltage through $K_{M}$ with an accuracy of $\sum_{\mathrm{k}=1}^{8} \varepsilon_{\text {sk }}(\mathrm{X}, \mathrm{Y}, \mathrm{Z})$, thus being added the voltages $U_{\text {shift }}(t, T)$ and $U_{R A N D}(t)$, which do not depend on the measured fields induction. As a result, the mathematical model of the systematic positioning error of the field measurement is proposed:

$$
P(D)=\frac{\Delta B_{x}}{B_{x}}(D)=\sum_{k=1}^{8} f_{x k}^{\prime} \cdot \Delta \chi_{k}+\varepsilon_{E M F}(D)=P_{1}(D)+P_{2}
$$

where: $P_{1}(D)=\varepsilon_{s 1}(D)+\varepsilon_{s 7}(D)+\varepsilon_{s 8}(D)+\varepsilon_{E M F}(D) ; P_{2}=\varepsilon_{s 3}+$ $\varepsilon_{s 2}+\varepsilon_{s 4}+\varepsilon_{s 5}+\varepsilon_{s 6} \neq f(D) ; D$ is the distance between the receiver and the transmitter.

The specificity of the positioning field measurements comes from Eq. 16; the error depends not only on the magnitude, but also on the distance $D$. This fact allows us to consider Eq. 16 as a function of the distance $P(D)$, which has an extremum. The minimization of $P(D)$ was performed by the dichotomy method under the condition:

$$
\mathrm{d} P(D) / \mathrm{d} D \rightarrow 0
$$

It is shown next that the condition (Eq. 17) runs at different distances $D$, depending also on the properties of the measuring system. A presented mathematical model of measurements of positioning field allowed to improve further the magnetic tracking theory as well as to explore the impact of the properties of receiver and analog-digital converters, the influence of distance, and the errors of mathematical description of the positioning field. The investigation of this model made it possible to create metrological ensuring of indoor-navigation theory and to formulate the requirements for mobile receiver.

\section{NUMERICAL SIMULATION}

Equation 3 can be solved by numerical methods, using the following iterative formula:

$$
\mathrm{X}_{\mathrm{i}}=\mathrm{X}_{\mathrm{i}-1}-\mathrm{STEP} \cdot \operatorname{grad}(\mathrm{CF})
$$

where: $\mathbf{X}=(x, y, z, \alpha, \beta, \gamma)$ is the vector of desired coordinates of movable receiver as already mentioned; $i$ is the number of iterations; STEP is the step of the iterative procedure; $\mathrm{CF}(\mathrm{X} \in \mathrm{R})=\sum_{\mathrm{n}=1}^{\mathrm{N}} \sum_{\mathrm{k}=1}^{\mathrm{K}}\left(\left({ }_{k n c}(\mathrm{X})-B_{k n m}\right)^{2}\right.$ is the functionality to minimize, being $n$ the number of windings in the source, $k$ the number of sensors in the receiver, $B_{k n c}$ the calculated values of induction for the assumed coordinates, and $B_{k n m}$ the measured values of induction.

Different ways to determine the step in Eq. 18 were applied, like a constant step or the steepest descent method. In addition, it was researched the method of separation of variables into linear and angular, and, for each type of coordinates, it was solved the system of equations.

To connect both positioning and measurement errors, Monte Carlo method is applied to investigate the transformation of random measurement errors of positioning field induction $\left(\Delta B_{N}\right)$ into computation errors $(\Delta \mathrm{X})$ of coordinates. To simulate the noise of $\Delta B_{N}$, centered signals from a random generator were added to induction measurements in each channel: $\mathrm{d} B_{x}(j)=K_{N} \times 2 \times(Y(1,1)-0.5), \mathrm{d} B_{y}(j)=K_{N} \times 2 \times(Y(1,2)-0.5)$, and $\mathrm{d} B_{z}(j)=K_{N} \times 2 \times(Y(1,3)-0.5)$, where: $K_{N}=T \times j$ is the growing scale of the random process amplitude, the same for all channels; $T$ is the rate of the amplitude growing; $Y=\operatorname{rand}(1,3)$ is the matrix of random uncorrelated numbers, with dimension $1 \times 3$ in scale $0-1$.

Adding the same random process at all measuring channels models, the impact of external disturbances is $\mathrm{d} B_{x}(j)=\mathrm{d} B_{y}(j)=\mathrm{d} B_{z}(j)=K_{N} \times 2 \times(Y(1,1)-0.5)$. It was investigated the dependence of RMS of computed coordinates $\left(\sigma_{x}\right)$ versus the RMS of input random process $\left(\sigma_{\text {Bmax }}\right)$. The transformation of multiplicative measurement errors was explored by the Monte Carlo method for coincidence of the real coordinates and initial approximation. In this case, the measured signals were multiplied by $K_{M}=1+0.01 \times j \times \varepsilon_{S}$, being $\varepsilon_{S}$ the limit value of the multiplicative measurement errors, and $j$ the point number at the interval $0-100$ with step 1 : $B_{x}(j)=B_{x}(0) \times K_{M} ; B_{y}(j)=B_{y}(0) \times K_{M} ; B_{z}(j)=B_{z}(0) \times K_{M}$.

The dependence was investigated on residual coordinate deviations

$$
\delta \mathrm{x}=\sqrt{\frac{1}{3} \cdot\left[\left(x-x_{r}\right)^{2}+\left(y-y_{r}\right)^{2}+\left(z-z_{r}\right)^{2}\right]}
$$

versus the increasing values of the multiplicative measurement error $\delta \mathbf{B}_{m}=f_{1}\left(j \times \varepsilon_{S}\right)$. For the study of transformation of the additive error component, it was suggested adding to the measured signals the following components: $B_{x}(j)=B_{x}(0)+0.01 \times i \times \varepsilon_{A} \times B_{x}(0), B_{y}(j)=B_{y}(0)+0.01 \times i \times$ $\varepsilon_{A} \times B_{y}(0)$, and $B_{z}(j)=B_{z}(0)+0.01 \times i \times \varepsilon_{A} \times B_{z}(0)$, being $\varepsilon_{A}$ the limit value of the additive measurement errors from Eq. 6 , $i$ the step number in the range of $0-100$ with step 1 , and $B_{x}(0)$ $\ldots B_{z}(0)$ the values of the vector component of the induction at the observation point. Here, the dependence of $\mathrm{d} x$ from Eq. 19 
was investigated in comparison with the increasing values of the additive measurement error $\delta \mathbf{B}_{m}=f_{1}\left(j \times \varepsilon_{S}\right)$.

\section{PHYSICAL MODELS}

At the final stage of investigation, in order to verify the created theory, the sample of active magnetic positioning system for targeting at a distance of up to $1 \mathrm{~m}$ was developed in the following composition: the movable receiver, consisted of 3 orthogonal Hall sensors (right part of Fig. 9); the source of the orthogonal fields with ferromagnetic core (left part of Fig. 9, Table 3); controller; computer with interface; and software to solve the task of active positioning in real time. The receiver's volume was $-27 \times 10^{-6} \mathrm{~m}^{3}$, and the distance between receiver and computer was $10 \mathrm{~m}$.

The objectives of the physical models study are:-metrological research of movable receiver (the impact of temperature and the instability of the supply voltage on the accuracy of the measurement); calibration of receiver's sensitivity and orientation; metrological research of positioning field source (accuracy of the field description, eddy current decay time constant, amendments mapping, and amplitude stability of pulse field over time); trial positioning of movable receiver (stability "at the point", positioning on the specified path, influence of the interior, and operation ranges score).

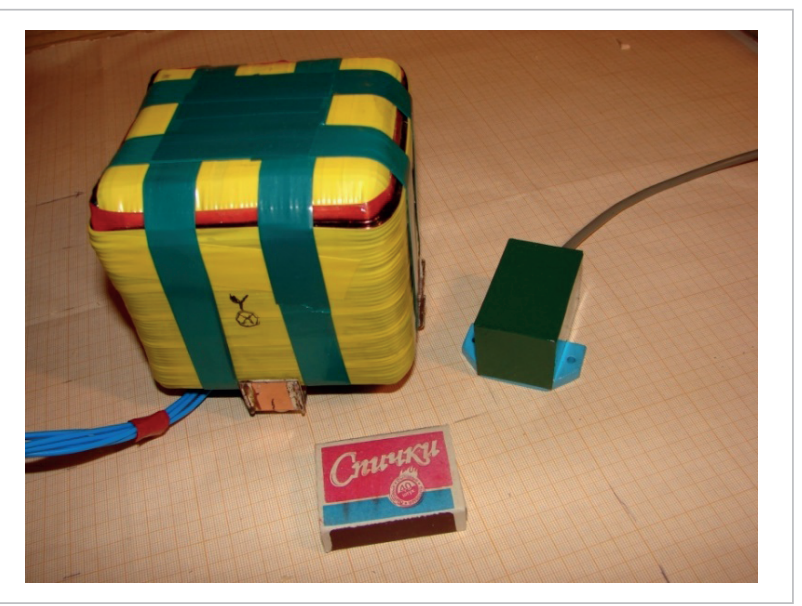

Figure 9. Mobile receiver (right) and the source of positioning field.

\section{RESULTS}

The accuracy of positioning field description was estimated by numerical and physical simulation. The estimates were compared for different models - a single thin frame and winding with
Table 3. Basic parameters of the positioning field source.

\begin{tabular}{|c|c|}
\hline Parameter & Value \\
\hline Weight & $6.8 \mathrm{kG}$ \\
\hline Dimensions & $\sim 100 \times 100 \times 100 \mathrm{~mm}$ \\
\hline Power consumption per winding & $<40 \mathrm{~W}$ \\
\hline Amplitude of excitation current & $8 \mathrm{~A}$ \\
\hline Source-receiver maximum distance & $0.75 \mathrm{~m}$ \\
\hline Step of field mapping & $30 \mathrm{~mm}$ \\
\hline $\begin{array}{c}\text { Induction value at maximum } \\
\text { distance }\end{array}$ & $0.1 \mathrm{Gauss}$ \\
\hline $\begin{array}{c}\text { Accuracy of field description } \\
\text { without core }\end{array}$ & $\leq 0.02 \%(\mathrm{D}>0.5 \mathrm{~m})$ \\
\hline Amendments to analytical model \\
due to core
\end{tabular}

ultimate square and different shapes. Table 3 shows the main parameters of the source for modes with and without core. For the trial mapping of source with core, it was selected the area in 1 quadrant, delimited by the following coordinates: $0.13<x<0.61$, $-0.51<y<-0.03$, and $-0.25<z<-0.13 \mathrm{~m}$. The number of cubic element is $N=15 \times 15 \times 3=675$, which contains $U=16 \times 16 \times 4=1,024$ grid nodes. Each node was identified by discrepancy of 3 axial components of the field induction, created by each of the 3 orthogonal windings of the source. The total number of measurements is $M=9,216$. The mapping of the source with ferromagnetic core showed that the amendments (Eq. 11) can be obtained as the difference between the measured and the calculated values for the corresponding nodes.

The maximum voltage at the source winding in rise or discharge was $<400 \mathrm{~V}$. Thus, to take into account individual features, inherent to the source with core, it is necessary the custom factory certification procedure by field mapping to get the array of amendments in respect to the model of "clean windings" from Eq. 9. The eddy current decay time constant in the source was experimentally estimated at the level of 
$55 \pm 5 \mu \mathrm{ks}$, which is much lesser than the discharge time constant $\tau$ from Eq. 12.

Numerical simulation of influence of eddy currents in the cockpit interior revealed that near the mobile receiver $\left(D_{2} \rightarrow 0\right)$ small items $(D<20 \mathrm{~mm})$ of electroconductive materials do not affect positioning accuracy. The measurements show that small electroconductive elements (fastening to $M_{8}$ ), located directly at a movable receiver, affect the results of the coordinates computation at a level of $\pm 0.1^{\circ}$ and $\pm 1 \mathrm{~mm}$. Ferromagnetic items in this mode are not affected at the size up to $M_{3}$. In accordance with Eq. 13, the conductive plate with thickness of $3 \mathrm{~mm}$ and area up to $S \leq 0.1 \mathrm{~m}^{2}$ does not affect the moving off $\left(D \geq 0.5 \mathrm{~m}\right.$ ) from the receiver. If the distance $D_{2}$ (see Fig. 7) is comparable with the size of the item $D_{2} \leq 2 r$, the influence of the electrically conductive element on the measured induction is at the level of $\Delta B / B \leq 1 \%$. Large ferromagnetic elements at the level of mass $m \approx 1 \mathrm{~kg}$ (like a gun) are affected on the distance $D \leq 0.3 \mathrm{~m}$, not being on the source-receiver line. Considering that the volume of ferromagnetic materials is much smaller than that of electrically conductive ones in the cockpit of a modern aircraft (and they are not situated between receiver and source), it can be assumed that the mapping of the cockpit may not be necessary. In addition, it is shown that, if not closer to the helmet's covering cabins, eddy currents do not affect the positioning accuracy.

The results of numerical simulation of measurement errors from Eq. 15 are shown in Table 4, where it can be seen that the total error of positioning field measurement has a minimal extremum at a distance $D=0.5 \mathrm{~m}$ which can match the original status of the helmet in the cockpit.

The numerical investigations of positioning error showed that, to obtain the error of linear coordinates calculation at a level $\Delta x= \pm 1 \mathrm{~mm}$ and at a distance $L=1 \mathrm{~m}$, the multiplicative error of magnetic induction measurement should be no worse than $\Delta K / K \approx \pm 0.5 \%$. In the middle of the movement zone, where the value of the total additive error is $\Delta B_{S}+\Delta B_{F} / B= \pm 0.3 \%$, it

Table 4. Total error of measurements at different distances from the source.

\begin{tabular}{c|c|c|c|}
\hline $\begin{array}{c}\text { Distance/errors } \\
{[\%]}\end{array}$ & $\begin{array}{c}\text { Multiplicative } \\
{\left[\varepsilon_{\mathrm{M}}\right]}\end{array}$ & $\begin{array}{c}\text { Additive } \\
{\left[\varepsilon_{\mathrm{A}}\right]}\end{array}$ & $\begin{array}{c}\text { Ramdom } \\
{\left[\varepsilon_{\mathrm{r}}\right]}\end{array}$ \\
\hline $\mathrm{D}=0.1 \mathrm{~m}$ & \pm 0.85 & \pm 1.325 & $\varepsilon_{r 1} \rightarrow 0$ \\
\hline $\mathrm{D}=0.5 \mathrm{~m}$ & \pm 0.85 & \pm 1.1 & $\varepsilon_{r 1} \leq \pm 0.1 \%$ \\
\hline $\mathrm{D}=1 \mathrm{~m}$ & \pm 0.85 & \pm 5.55 & $\varepsilon_{r 1} \leq \pm 0.3 \%$ \\
\hline
\end{tabular}

$\varepsilon_{M}=\varepsilon_{S 2}+\varepsilon_{S 4}+\varepsilon_{S 5}+\varepsilon_{S 6^{\prime}} \varepsilon_{A}=\varepsilon_{S 1}+\varepsilon_{S 3}+\varepsilon_{S 7}+\varepsilon_{E M F}$ can be supposed the accuracy of linear positioning at the level $\Delta x \approx \pm 2 \mathrm{~mm}$ or $\Delta x / x \approx 0.4 \%$. The error of the angles calculation is no worse than $\pm 0.3^{\circ}$ throughout the full range of operation and is almost independent of the distance. To obtain the RMS of angles $\sigma_{\varphi}=0.1^{\circ}$, it is necessary to have the RMS of magnetic induction measurement at the level $\sigma_{B}=10-20$ nTesla. In all cases, the maximum scores of errors are specified, so that they can be reduced to an exact task optimization.

According to the experimental research of the sample of mobile receiver, its own noise's RMS provides the signal-noise ratio no worse than $30 \mathrm{~dB}$. To work in a wide range of ambient temperatures (inherent aviation), it is offered a micro-thermostat for the helmet's receiver, which reduces the temperature variation of the Hall sensor off-set in 80 times.

The algorithm is proposed to take into account the instability of the supply voltage, which allowed to reach the error of the mobile receiver coordinates calculation not worse than $\Delta x= \pm 2 \mathrm{~mm}$ and $\Delta \varphi= \pm 0.5^{\circ}$ for variations of the supply voltage of receiver and source windings within $\pm 5 \%$. The investigation showed that, to obtain a requested precision of positioning, the mutual orientation sensors in mobile receiver should be determined with an accuracy no worse than $\pm 0.5^{\circ}$. The suggested algorithm for accounting of spatial sensors separation allowed to reduce the error of module calculation "near the source" from $\varepsilon_{s 2}=\left(B_{C}-B_{M}\right) / B_{C} \times 100 \%=6 \%$ to a value $< \pm 1.0 \%$.

The RMS of calculated coordinates of the stationary receiver was not worse than $\sigma_{X}=1 \mathrm{~mm}$ and $\sigma_{\varphi}=0.2^{\circ}$, using the calculation algorithm from Eq. 18 For the modeling of helmet's linear motion within the cockpit, it was studied the movement of the receiver along the straight line $y=k x+b(b=0, k=1)$; $z=$ constant on the surface XOY in the coreless source field. The orientation of the receiver remained unchanged during the movement. The investigation results are presented in Fig. 10, where linear coordinates are given in Fig. 10a and angles of orientation in Fig. 10b versus distance $\mathrm{D}=\sqrt{\mathrm{x}^{2}+\mathrm{y}^{2}+\mathrm{z}^{2}}$ from the source center.

It is seen the linear ramp-up of $X$ and $Y$ coordinates, which corresponds to the selected type of movement. The step between nearby points on the trajectory is not less than $50 \mathrm{~mm}$, which is provided by the convergence of the computing procedure. In the coreless field source, the motion of the receiver onto the circle trajectory on a horizontal plane XOY of aircraft was also studied, which simulates the mode of the target selection by turning the pilot's head. Figure 11 shows the coordinates calculated 


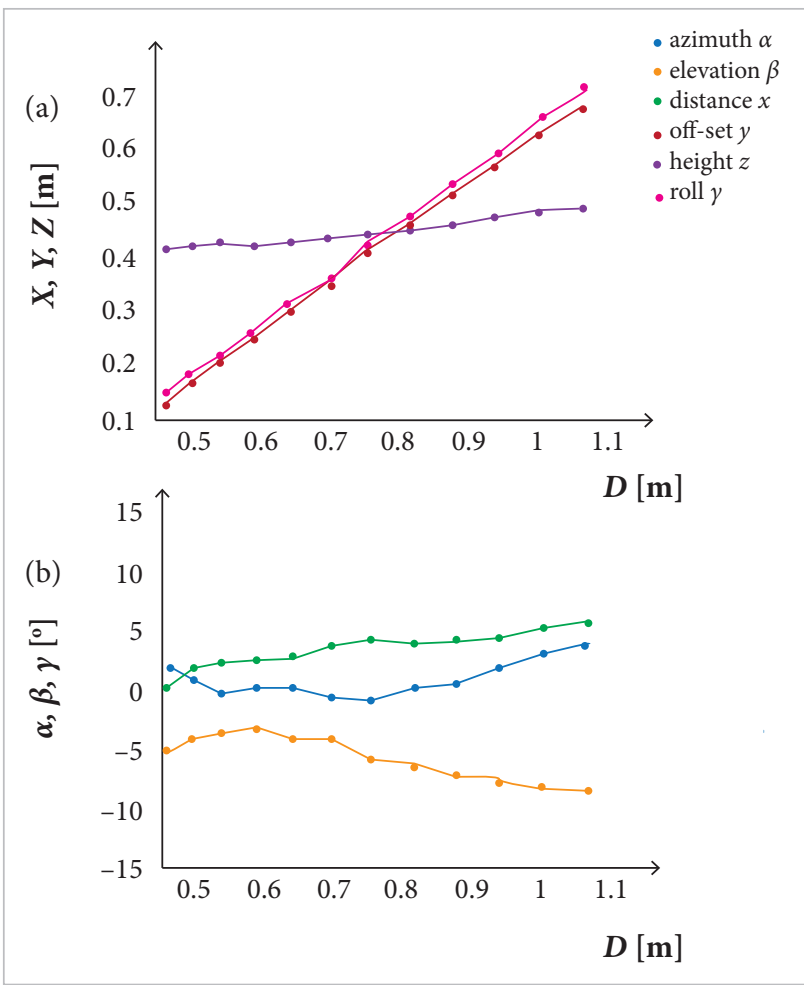

Figure 10. Motion along the straight line in field of coreless source. (a) Linear coordinates; (b) Angles of orientation.

from Eq. 18 for the mobile receiver moving around the $Z$ axis on the plane $X O Y$ along the circle trajectory in accordance with equation $\left(x-x_{0}\right)^{2}+\left(y-y_{0}\right)^{2}=R^{2}$, where $R=0.2 \mathrm{~m}$ and $\left(x_{0}, y_{0}\right)$ are the radius and the central coordinates of rotation, respectively.

In both cases, a 6-DOF positioning is seen. The systematic errors of the calculated coordinates associated with insufficient precision of movable receiver model calibration at the stage of the $1^{\text {st }}$ sample will be eliminated during the transition to the prototype through the R\&D phase. To reduce energy consumption of on-board cueing system, it was researched the source of positioning fields with both ferromagnetic core and map of amendments. Figure 12 shows the results of a trial positioning of the mobile receiver during a movement along the full circle in $X O Y$ plane around the $Z$ axis - in this case, we have $R=0.16 \mathrm{~m}$. The map of amendments was used, obtained by mapping of the source field in free space, as already described.

The systematic errors in this case may be explained by a lack of accuracy in the amendments maps at the stage of the HMCS's $1^{\text {st }}$ mockup sample. As a result of the

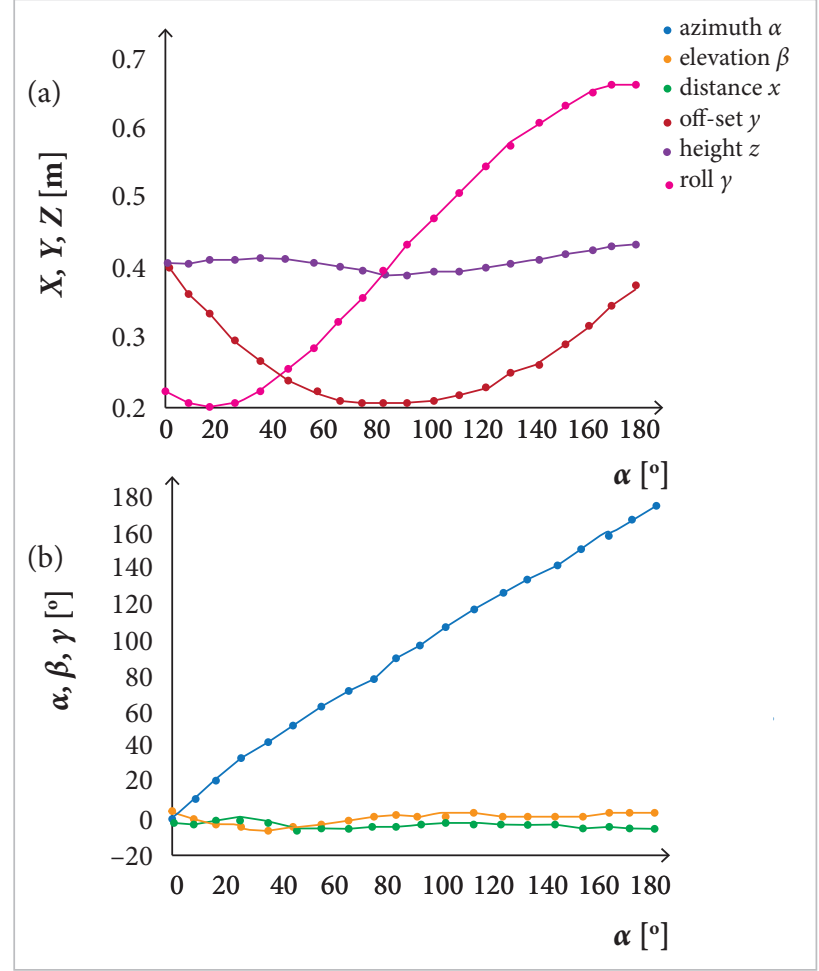

Figure 11. Traffic on the half-circle trajectory. (a) Linear coordinates; (b) Angles of orientation.

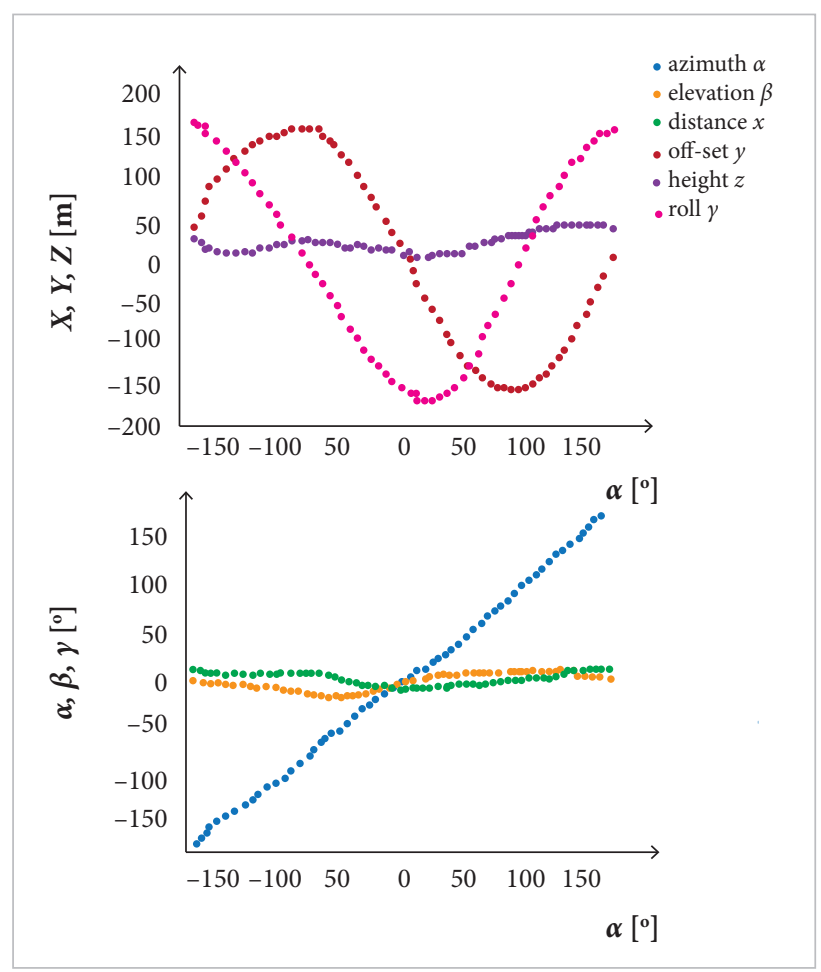

Figure 12. Movement on a full circle in the source field with core. (a) Linear coordinates relative to the center of rotation; (b) Orientation angles. 
transition to the R\&D phase, the accuracy in the amendments maps calculation will be increased. The investigations show that the created sample operates in full ranges of orientation angles of the receiver $-\alpha=\beta=\gamma= \pm 180^{\circ}$. The core allowed a 3.5 times increase in the induction of an external field of positioning at the same amplitude of the excitation current - or at the same time reduction current at the same magnitude of the measured induction. Power consumption is reduced by an order of magnitude.

\section{CONCLUSION}

The proposed concept is to build a new generation of HMCS on magnetic principle, based on a new way of positioning field organization. The new approach suggests the measurement of the positioning field induction on the basis of modern magnetometers for smartphones, with minimum dimensions, which eliminates the problem of weight and dimensions of the helmet-mounted receiver. As a result, it is shown the possibility to increase the update rate and accuracy of measurements and positioning, in order to reduce power consumption and dimensions of the proposed HMCS in comparison with prototype.

The investigations have shown that mapping the cockpit is not necessary in the application of a new method for positioning field organization, due to negligible effect of the interior. The physical experiments show the functioning of HMCS sample in full ranges of orientation angles. Created mathematical models allow to simulate error of descriptions and measurements of positioning field induction as well as positioning errors to select the optimal configuration of HMCS for a specific aircraft.

Thus, it is possible to figure out the next generation of HMCS with enhanced features: angles range - unlimited; angular accuracy $\leq \pm 0.1^{\circ}$; linear accuracy $\leq \pm 1 \mathrm{~mm}$; update rate $-200 \mathrm{~Hz}$; and influence of EMF - twice reduced.

The enterprising researches in this area are being continued in Russia, where more than 30 scientific articles on this subject were already published.

\section{REFERENCES}

Analog Devices (2016) Inertial measurement units; [accessed 2016 Aug 19]. http://www.analog.com/en/products/mems/isensormems-inertial-measurement-units.html

Asahi Kasei Corp. [2016] AK8975/AK8975 3-axis electronic compass; [accessed 2016 Aug 26]. http://www.asahi-kasei.co.jp/ asahi/en/

Ascension Technology Corporation (2016) 3D guidance; [accessed 2016 Aug 19]. http://www.ascension-tech.com/realtime/ virtualreality.php

BAE Systems (2016) Striker® II digital helmet-mounted display; [accessed 2016 Aug 19]. http://www.baesystems.com/en-uk/ product/striker-ii-digital-helmet-mounted-display

Bosh Sensortec (2016) 9-axis sensors; [accessed 2016 Aug 26] http://www.bosch-sensortec.com/en/homepage/products_3/9_ axis_sensors_5/9-axis_sensors

Blood EB, inventor; Device for quantitatively measuring the relative position and orientation of two bodies in the presence of metals utilizing direct current magnetic fields. 1989 Jul 18. Ascension. United States patent US 4849692 A.

Buganov VI (2016) KP "CDB" Arsenal — the improvement and creation of new opto-electronic aircraft equipment; [accessed 2016 Aug 19]. http://www.tinlib.ru/transport_i_aviacija/aviacija_i_vremja_2003 specvypusk/p12.php

Defencetalk.com (2007) Denel optronics head-tracker system for Eurofighter typhoon; [accessed 2016 Aug 19]. http://www. defencetalk.com/denel-optronics-head-tracker-system-for-eurofightertyphoon-12206/
Egli WH, Jeffrey MS, Weir EJ, inventors; Helmet metal mass compensation for helmet-mounted sighting system. 1983 Jul 26. Honeywell Inc. United States patent US 4394831 A.

Elbit Systems of America $(2006)$ Helmet Mounted Display and Sight Systems; [accessed 2016 Aug 19]. http://defense-update.com/ directory/elbit-hmd.htm

Elbit Systems of America® (2016) IHADSS; [accessed 2016 Aug 19]. http://www. elbitsystems-us.com/airborne-solutions/products-subsystems/helmet-mounted-systems/helicopter-helmets/ihadss

Ivensense® (2016) 9 axis motion tracking; [accessed 2016 Aug 22]. http://www.invensense.com/products/motion-tracking/9-axis/

Khalfin I, inventor; Method and apparatus for electromagnetic position and orientation tracking with distortion compensation employing a modulated signal. 2004 Jul 13. Polhemus, Inc. United States patent US6762600 B2.

Kopp C (1982) Heat-seeking missile guidance; [accessed 2016 Aug 19]. http://www.ausairpower.net/TE-IR-Guidance.html

Kopp C (1998) Helmet mounted sights and displays; [accessed 2016 Aug 19]. http://www. ausairpower.net/hmd-technology.html

Kuipers J, inventor; Object tracking and orientation determination means, system and process. 1975 Feb 25. United States patent US 3868565 A.

Lescourret $\mathrm{JL}$, inventor; Method of compensation of electromagnetic perturbations due to moving magnetic and conducting objects. 1997 Dec 2. Sextant Avionics. United States patent US 5694041 A.

Melzer JE, Moffitt KW (1997) Head-mounted displays: designing for the user. New York: McGraw-Hill. 
Merryman RFK (1994) Vista Sabre II: integration of helmet-mounted tracker/display and high off-boresight missile seeker into F-15 aircraft. Proc SPIE 2218:1-12. doi: 10.1117/12.177361

Polhemus (2016) Motion tracking overview; [accessed 2016 Aug 19]. http://www.polhemus.com/motion-tracking/overview/

Raab FH, inventor; Remote object position locater. 1977 Oct 18. The Austin Company. United States patent US 4054881 A.

Thales Group (2016) TopOwl® helmet-mounted sight and display for helicopters; [accessed 2016 Aug 19]. https://www.thalesgroup. com/en/worldwide/defence/topowlr-helmet-mounted-sight-anddisplay-helicopters

Thales Visionix, Inc. (2016) Pilot; [accessed 2016 Aug 19]. http:// www.thalesvisionix.com/pilot/
Zhelamskij MV, inventor; Method to define the linear position and orientation of movable object. 2011 Mar 10. Russia patent 2413957 RU.

Zhelamskij MV (2014a) Features of the construction of a positioning field for local navigation in enclosed spaces. Meas Tech 57(7):791799. doi: 10.1007/s11018-014-0538-5

Zhelamskij MV (2014b) Features of the measurement of a local positioning magnetic field at short distances. Meas Tech 57(9):10321040. doi: 10.1007/s11018-014-0577-y

Zhelamskij MV (2015) The positioning of mobile objects in local magnetic field: theory and practice. Saarbrucken: Palmarium Academic Publishing. In Russian. 